\title{
Beam Shaping for CARS Measurements in Turbulent Environments
}

\author{
Gaetano Magnotti ${ }^{1}$, Andrew D. Cutler ${ }^{2}$ \\ The George Washington University \\ Newport News VA 23602, USA \\ Paul M. Danehy ${ }^{3}$ \\ NASA Langley Research Center \\ Hampton VA 23681, USA
}

\begin{abstract}
This paper describes a new technique to mitigate the effect of beam steering on CARS measurements in turbulent, variable density environments. The new approach combines Planar BOXCARS phase-matching with elliptical shaping of one of the beams to generate a signal insensitive to beam steering, while keeping the same spatial resolution. Numerical and experimental results are provided to demonstrate the effectiveness of this approach. One set of experiments investigated the effect of beam shaping in the presence of a controlled and well quantified displacement of the beams at the focal plane. Another set of experiments, more qualitative, proved the effectiveness of the technique in the presence of severe beam steering due to turbulence.
\end{abstract}

\section{Nomenclature}

$P_{C A R S}=$ CARS signal power

$I \quad=$ irradiance of the laser beam

$c \quad=$ speed of light

$\omega_{\text {CARS }}=$ CARS signal frequency

$\chi^{(3)}=$ third order susceptibility

$\sigma_{x} \quad=$ beam width along $x(1 / \mathrm{e}$ of the electric field $)$

$\sigma_{y} \quad=$ beam width along $y(1 / \mathrm{e}$ of the electric field $)$

$\alpha=$ angle between the beam direction and the direction of the CARS signal

$d \quad=$ full width half max (FWHM) of the beam irradiance

$B D=$ beam displacement coefficient (Eq. 5)

$D \quad=$ beam diffusion coefficient (Eq.8)

\section{Introduction}

$\mathrm{Q}$ UANTITATIVE measurements in a scramjet engine are particularly challenging because of the harsh testing environment. Any material probe inserted in the flow, if it were not destroyed, would strongly perturb the flow. Coherent Anti-Stokes Raman Spectroscopy (CARS) is a non-intrusive laser-based measurement technique that has been successfully implemented by several researchers to measure temperature and species concentrations in scramjet combustors. ${ }^{1,2,3}$ At NASA Langley Research Center, the Dual Pump CARS technique, originally developed by Lucht, ${ }^{4}$ was successfully used to measure temperature, $\mathrm{N}_{2}, \mathrm{O}_{2}$ and $\mathrm{H}_{2}$ concentration in scramjet engines. ${ }^{1,5}$ Recently, measurements in a large, supersonic combusting, axis-symmetric free jet, were performed. ${ }^{6,7}$ Test conditions were more challenging than in a ducted flow because of large density gradients, turbulence and vibration levels, resulting in large variations of the signal to noise ratio, and low data yield. ${ }^{8}$

\footnotetext{
${ }^{1}$ Graduate Student, AIAA Student Member

${ }^{2}$ Professor, Mechanical and Aerospace Engineering Department, 1 Old Oyster Point Road, Suite 200. Associate Fellow AIAA

${ }^{3}$ Research Scientist, Advanced Sensing and Optical Measurement Branch, MS 493, Associate Fellow AIAA 
CARS is a third order, non-linear optical measurement technique, in which three beams are focused and overlapped at the measurement volume, where they interact and generate a coherent signal containing the Raman spectrum of the probed species. Tens of micrometers of displacement are sufficient to prevent the beams from fully overlapping, causing a reduction in the signal-to-noise ratio. Turbulence produces rapid variations in space and time of the refractive index; larger eddies, with size comparable to the beam diameter, produce random motions of the beam focus, and smaller eddies diffuse it. Mechanical vibrations may displace the beams, with similar consequences on beams overlap. The magnitude of these effects is often tolerable for ducted flow such as a scramjet combustor but becomes critical for large free jets or high pressure combustors. ${ }^{9,10}$ In Ref. 8 Tedder et al. identify lack of beam overlap as main cause of low signal intensity and data yield in their measurements in a supersonic free jet.

The objective of this paper is to investigate a new technique to reduce the sensitivity of CARS to beam steering and vibrations. This new approach combines elliptical beam shaping and planar BOXCARS to achieve this goal while leaving the length of measurement volume unaltered. The new technique is compared to previous methodologies used for measurements of large turbulent flows. Advantages and limits of the technique are investigated numerically and experimentally.

\section{Beam steering mitigation technique}

In order to obtain a strong CARS signal, three incident beams have to be aligned so that the signal generated at some point will be in phase with the signal generated at subsequent points. Several phase matching strategies have been devised to satisfy the phase matching requirements while maintaining adequate spatial resolution. In the presence of beam steering some phase matching geometries are more robust than others. In USED CARS the pump beam is donut shaped and the Stokes beam is concentric to the pump. Because of the concentric geometry and of the common propagation axis of the beams, beam steering and vibrations have less effect on the overlap at the measurement volume. Eckbreth ${ }^{11}$ reports measurements in a jet engine exhaust using this approach, but the spatial resolution is $0.1 \mathrm{~mm}$ in diameter and $50 \mathrm{~mm}$ long, thirty times longer than in Refs. 1,5 and 6. In most applications a measurement volume length of $1 \mathrm{~mm}$ or less is required, strongly limiting the applicability of USED CARS.

Folded BOXCARS was used to collect the data in the large free jet of Refs. 6-8. This is a 3-dimensional arrangement that allows good spatial resolution and signal strength. This phase matching strategy is prone to be affected by beam steering; each beam travels through a different path, encountering different turbulence structures, and thus is refracted differently.

Planar BOXCARS (Figure 1) has all the beams travelling in the same plane. The phase matching condition requires that two of the three beams are almost superimposed as shown in Figure 1. In our system, two dye laser beams (the red broadband Stokes laser and the yellow narrow-band pump laser) are overlapped, and the CARS signal beam (in blue) is superimposed with the green pump beam. Dichroic mirrors are used to combine the two dye laser beams and to separate the signal from the green beam. The beams are crossed at their respective waists (which are forced to be

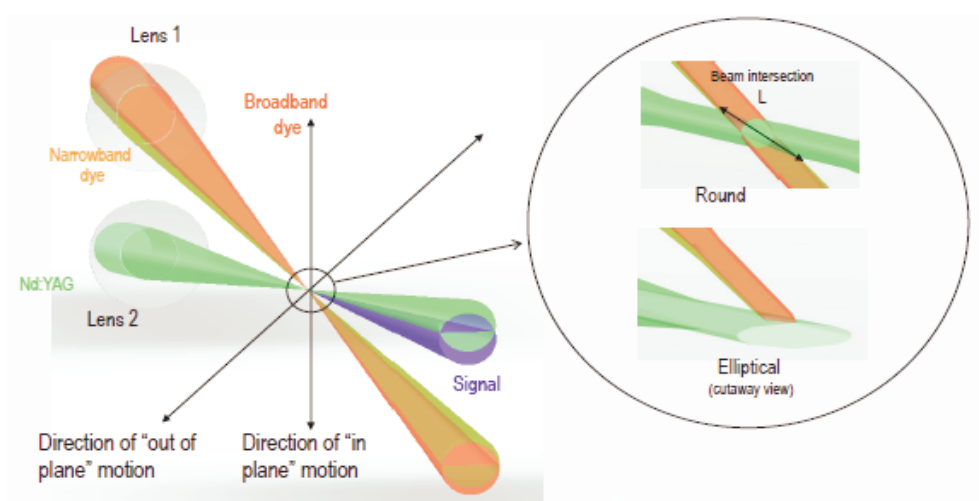

Figure 1. Planar BOXCARS phase matching geometry. In detail the beams at the intersection, for both round and elliptic green beam coincident), at the "focal plane". Signal is generated at the beam intersection, a region that resembles a very elongated ellipsoid with maximum cross section diameter determined by the smallest beam. The length of the probe volume is determined by the angle between the beams and by the beam diameters at the focal plane. ${ }^{12}$ The main disadvantage with respect to "folded" BOXCARS is the requirement of dichroic mirrors to combine and separate beams. The advantage is that the superposition of two beams ensures that they remain overlapped at the focus, even in the presence of beam steering, where in folded BOXCARS all three beams can move independently. Loss of signal will occur if the two superimposed beams do not overlap with the other beam. Beam displacement in the plane of the beams ("in-plane") does not prevent their overlap, but it shifts the crossing point away from the common focal plane. The resulting lower irradiance at the crossing plane causes a small reduction of the signal 
level. However, just hundreds of micrometers of displacement in the direction orthogonal to the plane ("out-ofplane") are sufficient to prevent overlapping and reduce the signal by few orders of magnitude.

An elliptical green beam, with the major axis in the direction orthogonal to the plane of the beams as shown in the detail of Figure 1, would allow beams to overlap for larger displacement out-of-plane than a round beam. Thus an elliptical beam in the planar BOXCARS geometry will generate a signal more insensitive to beam steering, but weaker because of the decreased irradiance, since the same energy is now spread across a larger area. Often in CARS setups the beam energy is limited by saturation conditions at the measurement volume and excess laser energy is not used. When shaping the beam, this energy can be used to keep the irradiance at the focus constant, preventing any signal loss. From geometric considerations ${ }^{12}$ the length of the interaction region is determined by the beam diameters in the plane of the beams, and does not depend on the diameter orthogonal to the plane. The diameter of the interaction region is given by the diameter of the smallest beam. Although the length of the measurement volume is not affected, with this technique uncertainty is added to the location of the measurement volume as in USED CARS.

\section{Numerical results}

In this section we evaluate numerically the CARS signal strength and probe volume length for planar BOXCARS when beam shaping is implemented and compare the results to what is obtained when using round beams.

The total CARS signal can be expressed as ${ }^{12,13}$

$$
P_{C A R S}=\frac{16 \pi^{4} \omega_{C A R S}^{4}}{c^{4}} \iint\left|\int\left(I_{g} I_{y} I_{r}\right)^{1 / 2} \chi^{(3)} d z\right|^{2} d x \cdot d y
$$

We introduce a Cartesian reference system with origin at the crossing point of the three beams; the $z$ axis is in the direction of the CARS signal beam, the $x$ axis is in the plane of the beams and orthogonal to $z$, and the $y$ axis is orthogonal to $x$ and $z$. Some simplifying assumptions have been made: each beam's irradiance distribution is approximated to a bivariate normal with null covariance:

$$
I(x, y, z)=\frac{P}{2 \pi \sigma_{x} \sigma_{y}} \exp \left\{-\frac{1}{2}\left[\frac{\left(x-\mu_{x}\right)^{2}}{\sigma_{x}^{2}}+\frac{\left(y-\mu_{y}\right)^{2}}{\sigma_{y}^{2}}\right]\right\}
$$

The parameters in the formula are function of the $z$ coordinate; $\mu_{y}=0$ for all three beams because the beams are coplanar; $\mu_{x}=z \cdot \tan \alpha$,

where $\alpha$ is the angle between the direction of the beam and the $z$ axis. The evolution along $z$ of $\sigma$ is approximated by the Gaussian beam relation ${ }^{14}$

$$
\sigma=\sigma_{0} \cdot \sqrt{1+\frac{z^{2}}{z_{R}^{2}}}
$$

where $\sigma_{0}$ is one half of the beam radius at the focal plane and $z_{R}$ is the Rayleigh range. The parameters $P, \sigma_{x}, \sigma_{y}$, and $\alpha$, are different for each beam. The length of the measurement volume is defined as the distance

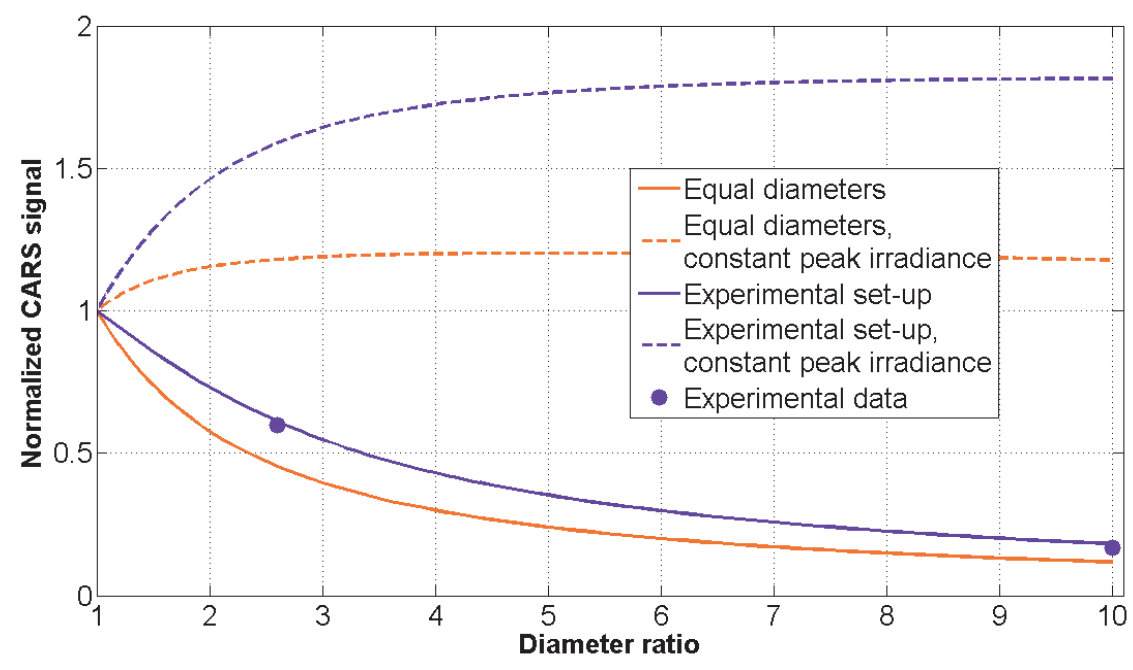

Figure 2. Normalized CARS signal as a function of the diameter ratio of the green beam. Results are normalized dividing by the values obtained for round beam. Blue dots are experimental results.

along $z$ over which the signal goes from $5 \%$ to $95 \%$. The integral is computed numerically on a grid of $50 \times 50 \times$ 100 cells. Doubling the number of points in each direction produces differences below $0.5 \%$ in the signal and measurement volume length.

Figure 2 shows a plot of the CARS signal as a function of the diameter ratio of the green beam obtained assuming a full width half maximum (FWHM) diameter of 50 microns for all the beams (except that the major 
diameter of the elliptical green beam is larger). The angle $\alpha$ is 4.5, 4.3 and -0.18 degrees, for the red, yellow and green beam respectively, conforming to phase matching requirements. The results are normalized by dividing by the values obtained for round green beam, so that they are independent of the beam irradiances. If the laser energy is kept constant the CARS signal drops with diameter ratio, but less than linearly. This is due to a higher irradiance in the region of the green beam that interacts with the other beams. If the peak laser irradiance is kept constant, then the signal increases up to 19\% for the 10:1 ratio. The results shown in Figure 2 are almost independent of the beam crossing angle and are only weakly dependent on the beam diameter if it is equal for all the beams. In the experimental setup described in Section IV the FWHM are about 40, and 100 and 120 microns respectively for the green, the narrowband and the broadband dye laser beams. The blue curves in Figure 2 are computed for the unequal beam diameters used in our experimental setup. As a consequence of the larger beam diameter for the two dye lasers, the signal strength decreases more slowly with ellipticity. When keeping the peak irradiance constant the difference is even larger, with a signal for a 5:1 diameter ratio that is $76 \%$ higher than was obtained for a round beam. The length of the probe volume does not depend on the diameter ratio.

The numerical results allowed us to characterize the effect of the beam shaping on CARS signal for perfect beam overlap, providing a baseline for the behavior in the presence of beam steering that is investigated experimentally in the next section.

\section{Experimental results}

\section{A. Experimental setup}

The beam steering mitigation technique has been tested using an updated version of the Dual Pump CARS system described in Ref. 6. An injection seeded pulsed Nd:YAG laser (Spectra Physics Quanta Ray Pro-350) frequency-doubled to $532 \mathrm{~nm}$, a narrow band dye laser (Spectra Physics PDL-2) centered at $551 \mathrm{~nm}$ and a homebuilt broadband dye laser centered at $603 \mathrm{~nm}$ with a FWHM of $10 \mathrm{~nm}$, are used to generate the CARS signal. The pulse width of the lasers is $8 \mathrm{~ns}$, the repetition rate $20 \mathrm{~Hz}$. The output of the Nd:YAG laser, approximately $900 \mathrm{~mJ}$, is divided three-ways: $170 \mathrm{~mJ}$ to pump the broadband dye laser, $415 \mathrm{~mJ}$ for the narrowband dye laser, and $280 \mathrm{~mJ}$ are left for the CARS green beam. The two dye laser beams are combined using a dichroic mirror. The three lasers are mounted on a mobile cart and the beams are relayed to the measurement volume through several mirrors. A combination of half wave plate and polarizing cube beam-splitter is used to finely tune the power of the green beam by reflecting undesired energy to a beam dump. This feature is used to avoid optical breakdown when a round beam is used and maintain constant beam peak irradiance when the diameter ratio is modified. With a round beam the green beam energy is limited to $68 \mathrm{~mJ}$, therefore up to $4: 1$ ratio is possible with our system while keeping the peak irradiance constant. The beams are focused by two $60 \mathrm{~cm}$ focal length spherical lenses, one for the superimposed dye lasers, and the other for the green beam. The energies of the red and yellow laser beams at the measurement
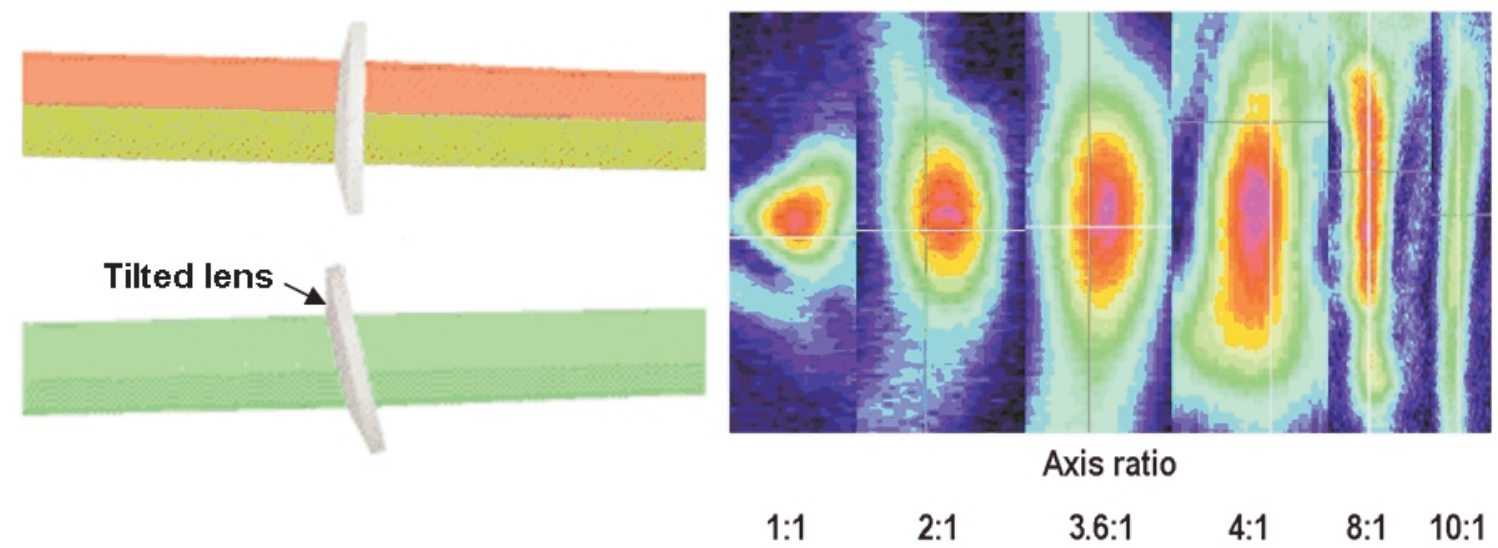

a)

b)

Figure 3. a) Beam shaping by tilting the lens. b) False color images of the green beam at the horizontal focal plane, for different lens tilting angles; beam size along minor axis is the same for all the images but the magnification is different.

volume are approximately $20 \mathrm{~mJ}$ and $50 \mathrm{~mJ}$. At the measurement volume they interact and generate the CARS signal, a coherent laser-like beam containing a broad CARS spectrum centered at $490 \mathrm{~nm}$. A series of dichroic 
mirrors and filters is used to separate the signal from the green beam. The signal is then focused by two cylindrical lenses on the input slit of a one meter spectrometer with a 2400 grooves $/ \mathrm{mm}$ grating. The CARS signal is dispersed by the spectrometer and recorded on a CCD camera. A beam viewing system is used to image the beams at the focal plane. ${ }^{15}$ The system consists of two wedged glass plates that collect a small portion of the three beams after the measurement volume, an achromatic lens that refocuses the beams and a microscope that magnifies the image on a CCD camera.

Elliptical beam shaping is easily achieved by tilting the focusing lens of the green beam as shown in Figure 3a. When the direction of the beam is not parallel to the lens axis, astigmatism occurs; therefore the in-plane (horizontal) and out-of-plane (vertical) foci occur at different locations along the path. (Note that in the experiment the plane of the beams is horizontal, unlike Figure 1 where it is vertical.) The CARS beams intersect at the in-plane focus. When the lens is rotated the in-plane focus is unchanged while the beam diameter at this focus increases exponentially out-of-plane.

Figure $3 \mathrm{~b}$ shows pictures of the green beam taken at the in-plane focus for several tilting angles of the lens and Figure 4 shows the in-plane and out-of-plane FWHM diameter of the beam as function of the tilting angle. Small tilt angles are sufficient to obtain a large diameter ratio. In our set-up the spherical lens used to focus the green beam is

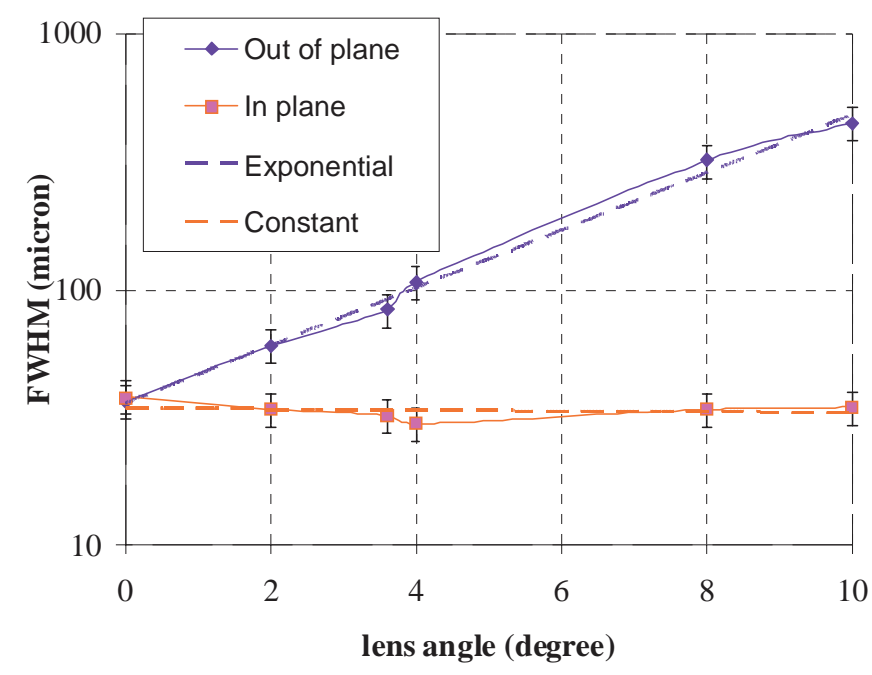

Figure 4. Full width half maximum diameter of the green beam at the focus point, as function of the lens tilting angle. Note logarithmic vertical axis mounted on a rotation stage with axis of rotation orthogonal to the plane of the beams. A translation stage is used to shift the in-plane focus of the green beam along the beam axis to the focus of the dye beams. With this setup, the diameter ratio of the beam can be varied continuously and no additional lenses are required. A pair of cylindrical lenses could have alternately been used to achieve a similar effect, had a single lens been used to focus and cross all three beams.

\section{B. Beam Displacement experiment}

Two sets of experiments have been performed to characterize the efficacy of this technique. In the first set of experiments the two superimposed beams are intentionally misaligned using adjustments on the last mirror before the focusing lens. The displacement is monitored through the beam viewing system. We introduce a dimensionless beam displacement coefficient $(B D)$ to quantify the effect of beam steering on the beam overlapping. We define it as

$$
B D=\left(B D_{x}, B D_{y}\right)=\left(\frac{l_{x}}{d}, \frac{l_{y}}{d}\right)
$$

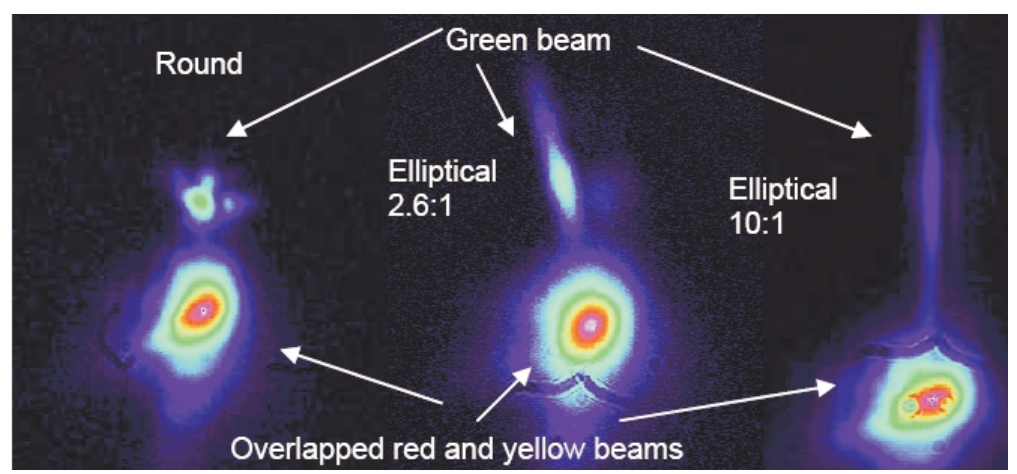

Figure 5. Three beams (shown in false colors) at focal plane for circular and elliptical configuration; beam size along minor axis the same for all green beams (orientation of beams is rotated 90 degrees compared to Fig. 1)

where $d$ is the yellow beam diameter at FWHM, $l$ is the average distance between the centroids of the red/yellow and green beams at the focal plane, and the subscript $x$ or $y$ indicates that the quantity is measured in-plane or out-ofplane. Our goal is to design a system to provide a measurable CARS signal even in flows with high $B D$. The coefficient is determined experimentally using the beam viewing system described in the previous section. Three diameter ratios have been considered for this experiment: 1:1, 2.6:1 and 10:1. Figure 5 shows the green beams for 
the three diameter ratios, and the overlapped yellow and red beams; note that the green beam and the overlapped red and yellow beams have been intentionally separated to make them distinguishable.

With the round green beam in a fixed location, the superimposed red and yellow beams were moved back and forth in-plane and then back and forth out-of-plane (see Fig. 1). Ambient air spectra were collected at each position. The area under each collected spectrum was integrated to obtain the signal. The normalized signal is the average signal over 1000 shots, divided by the peak average signal

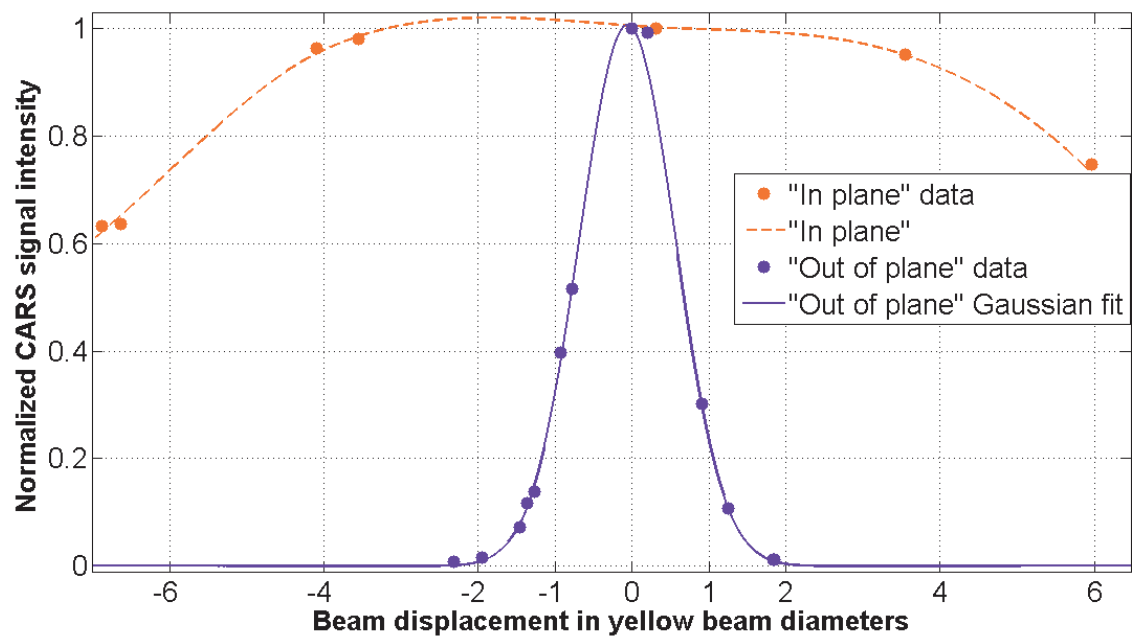

Figure 6. Sensitivity of the CARS signal to displacement of the overlapped yellow and red beams relative to the round green beam; signal normalized by the peak signal

which occurs at $B D=0$. The normalized signal plotted as a function of beam displacement is shown in Figure 6. Movement in-plane shifts the crossing point, so the beams are no longer crossing at the focal plane. The curve has a flat top because the beams stay focused for a small distance and then the curve slowly drops as the beam diameters at the intersection increase and irradiances decrease. Power is proportional to the square of the probe volume length, therefore this effect, although small, is noticeable in the curve. Movement out-of-plane causes a rapid drop in the signal level because the beams no longer overlap. Where a displacement of $4 B D$ in-plane decreases the signal level by less than $10 \%$, a displacement of $1 B D$ out-of-plane is sufficient to drop the energy to one third. The data follow a Gaussian curve with a FWHM of $1.34 B D$.

The effects of out-of-plane displacement with elliptical green beams of three different diameter ratios are shown in Figure 7. For beams crossing at the focal plane $\left(B D_{y}=0\right)$, the signal with the elliptical beam is $60 \%$ (2.6:1 ratio) and $16.7 \%$ $(10: 1$ ratio $)$ of the signal achieved with a round beam, because of the decreased irradiance at the focal plane. The values are slightly lower than what expected from numerical calculations $(61 \%$ and $18.2 \%$ as shown in Figure 2), and the difference is attributed to the beams not being perfectly Gaussian. The FWHM of the Gaussian fit of the data is $1.4 B D$

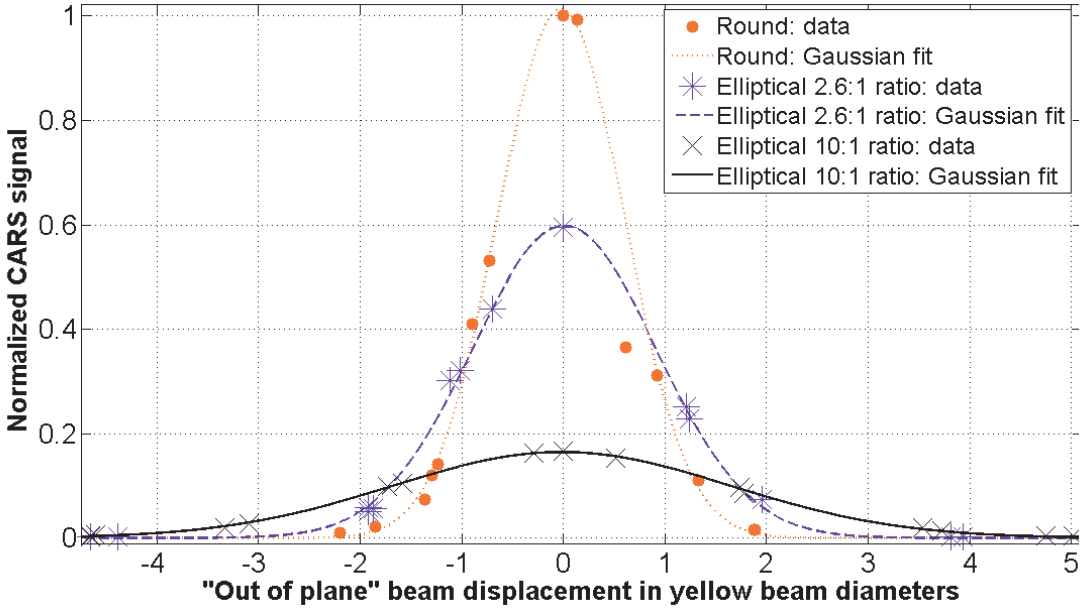

Figure 7. Sensitivity to out-of-plane beam displacement for round and elliptical green beam; the signal is normalized by dividing by the peak signal for the round green beam for the round beam, increases to $2.2 B D$ when using a 2.6 diameter ratio, and to $3.8 B D$ for the $10: 1$ ratio. This is evidence of the effectiveness of this strategy against beam displacement. The plot suggests that in the presence of severe beam steering, an elliptical beam can produce a stronger signal than a round beam. For $B D_{y}>0.7$, the 2.6 diameter ratio beam produces a stronger signal than a round, where a 10:1 diameter ratio becomes more efficient for $B D_{y}>2$. 
Figure 8 shows the same data points of Figure 7, normalized by the peak signal for that diameter ratio. This applies to a situation where the peak irradiance of the green beam is kept constant as the diameter ratio is changed. If the irradiance is limited by saturation effects, such as optical breakdown, stimulated Raman pumping or the Stark effect, the beam energy can be adjusted to keep the peak irradiance of the shaped beam constant. With our current configuration we were limited by breakdown, and only $25 \%$ of the total green energy could be used when the beam was round. When peak irradiance is kept constant the elliptical beam provides a signal that is stronger and more insensitive to beam steering than the signal generated using round beams. For example, a $B D_{y}=2$ reduces the signal level by two orders of magnitude when a round beam is used, one order of magnitude when an elliptical beam of diameter ratio of 2.6 is employed, and only $40 \%$ using a 10:1 ratio.

Figure 9 shows the CARS signal obtained combining in-plane and out-of-plane displacement, when using an elliptical green beam with a 5:1 diameter ratio. Each curve was obtained by varying the out-of-plane

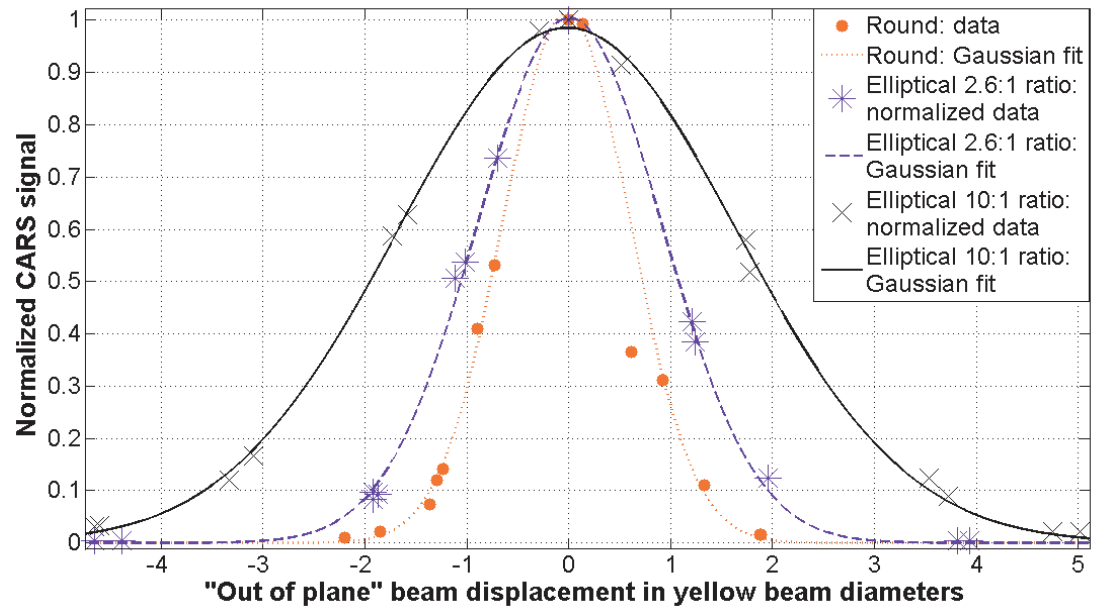

Figure 8. Sensitivity to out-of-plane beam displacement for round and elliptical green beam; the signal is normalized dividing by the peak signal

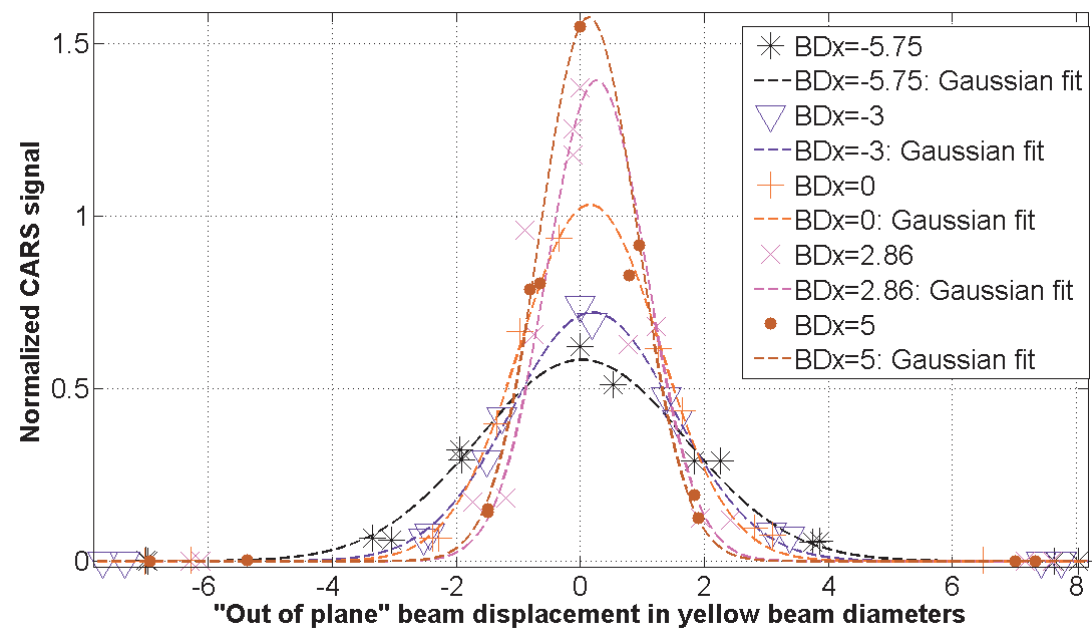

Figure 9. Sensitivity to combined out-of-plane and in-plane displacement for a green elliptical beam displacement $B D_{y}$ for a fixed amount of in-plane displacement $B D_{x}$. The signal is normalized by the signal when the beams are crossed at the focal plane $\left(B D_{x}=B D_{y}=0\right)$. Figure 9 shows that, for $B D_{x}<0$, the signal is weaker but more insensitive to out-of-plane displacements. Unexpectedly, for $B D_{x}>0$ the signal is stronger but more sensitive to out-of-plane motion.

Figure 10 helps explain this behaviour. Inplane displacement shifts the crossing point away from the focal plane (the location of the red, yellow and green in-plane foci). Figure 10 shows pictures of the green beam at the crossing plane, for the same values of in-plane displacement as plotted in Figure 9. Negative values of $B D_{x}$ shift the crossing point toward the focusing lens, therefore there is a small increase in the green major diameter and an increase in the green minor

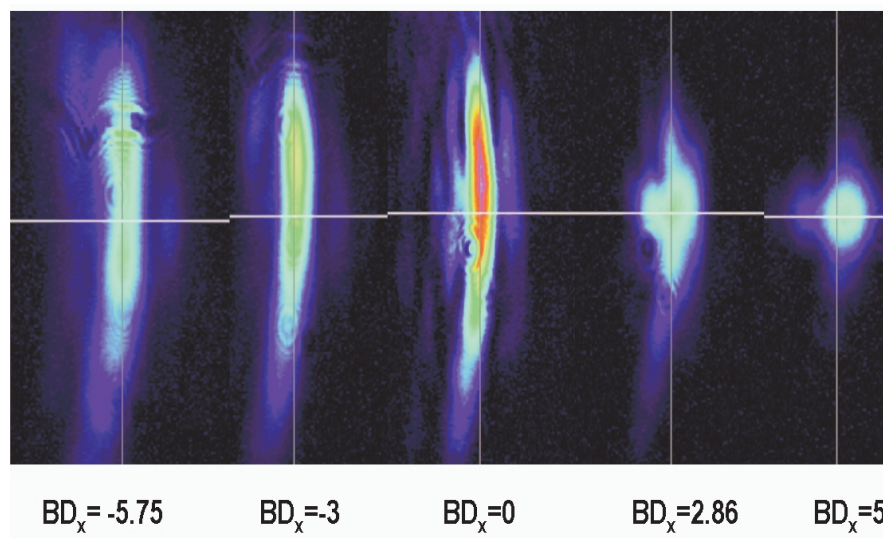

Figure 10. Green beam at the intersection with the yellow and red laser for several values of in-plane displacement

American Institute of Aeronautics and Astronautics 
diameter and in the red and yellow diameters. Larger beam waists produce a weaker CARS signal that is more insensitive to out-of-plane beam displacement as shown in Figure 9. Positive in-plane displacement moves the crossing point toward the out-of-plane focus, therefore the major diameter decreases and the minor diameter increases. For $B D_{x}>0$ the CARS signal is stronger, because of the increased irradiance of the green beam, but is more sensitive to out-of-plane displacement because of the reduced size of the green beam in the out-of-plane directions. These effects do not compromise the usefulness of beam shaping.

The results presented above depend on the diameters of the beams. Figure 11 shows results obtained for the round beam and the 8:1 elliptical beam, when the beam waist diameters are 50, 60 and 120 microns respectively for the green, yellow and red lasers. The FWHM of the Gaussian fit for the round beam is $1.42 B D$ where for the elliptical beam is $8.1 \mathrm{BD}$.

The dotted lines in Figure 11 are results obtained numerically using the algortihm of Section III, and assuming

$$
\mu_{y}=B D_{y} d
$$

for the red and yellow beam. The numerical results predict a

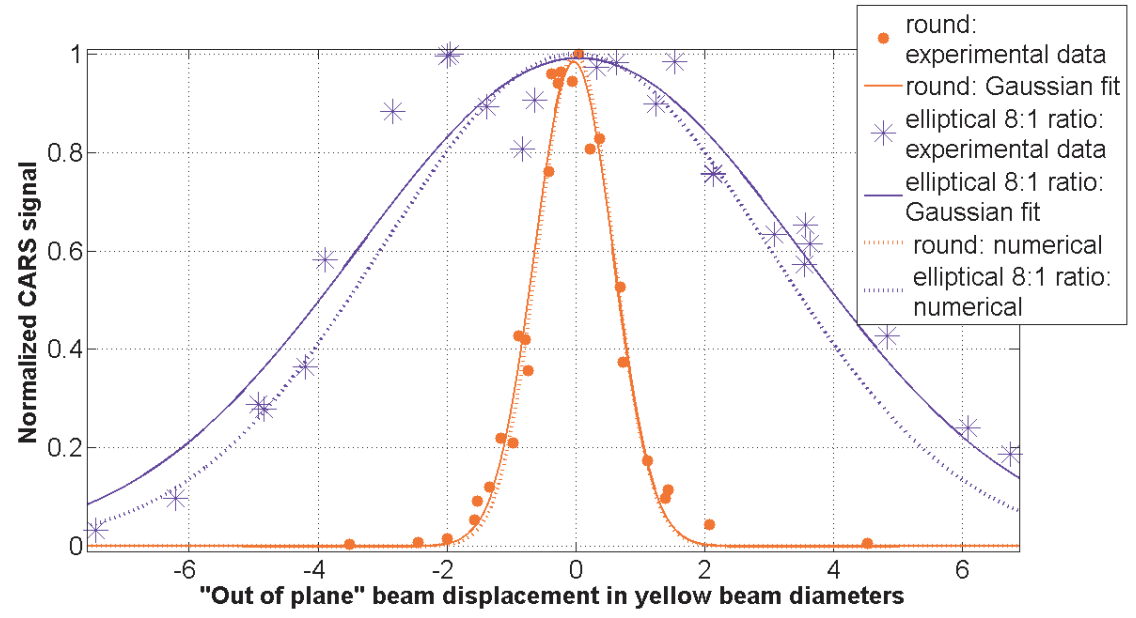

Figure 11. Sensitivity to out-of-plane beam displacement for round and elliptical green beam; the signal is normalized dividing by the peak signal. Dotted curves are obtained numerically using the algorithm of Section III

FWHM of $1.37 B D$ for the round beam (in agreement with the Gaussian fit to the experiment), and 7.1 $B D$ for the elliptical beam (12.3\% narrower). The validated numerical algorithm can be used to estimate the normalized CARS signal for any combination of out-of-plane beam displacement, beam diameters and green beam diameter ratio.

The beam displacement experiment provides quantitative results which prove that the technique makes the CARS signal more insensitive to misalignment. The results can be used as guideline to select an optimum diameter ratio for the green beam in the presence of strong vibrations.

\section{Turbulence effects experiment}

Beam refraction caused by turbulence in variable density flows is a more complex problem than misalignment due to vibrations. Two effects generally coexist: beam steering and beam diffusion. In these flows, density, and therefore index of refraction is not uniform either in space or time. Turbulent structures smaller than the beam refract some parts of the beam differently than others, resulting in random alterations of the beam irradiance profile at the focus. Larger turbulent structures are responsible for the random steering of the beam. Severe refraction effects are generally observed in large supersonic free jets ${ }^{8,10}$ or the exhaust of jet engines, ${ }^{11}$ because of long

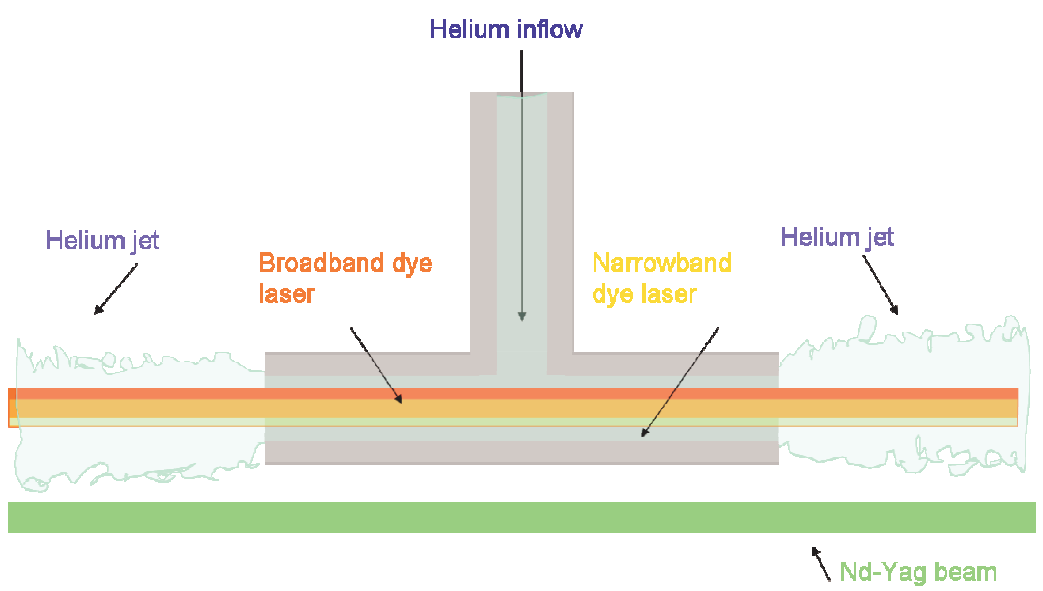

Figure 12. Schematic of the helium jet for beam steering production

beam paths, large density gradients and high levels of turbulence. Replicating such flows in the laboratory was not feasible, so we limited our effort to simulate their effect on the beams. Variations in the index of refraction can be obtained through changes either in pressure, temperature or gas composition. Helium has a very low refractive index 
in comparison to air; the difference in index of refraction between helium and air is roughly the same as the difference between air at $2300 \mathrm{~K}$ and ambient air.
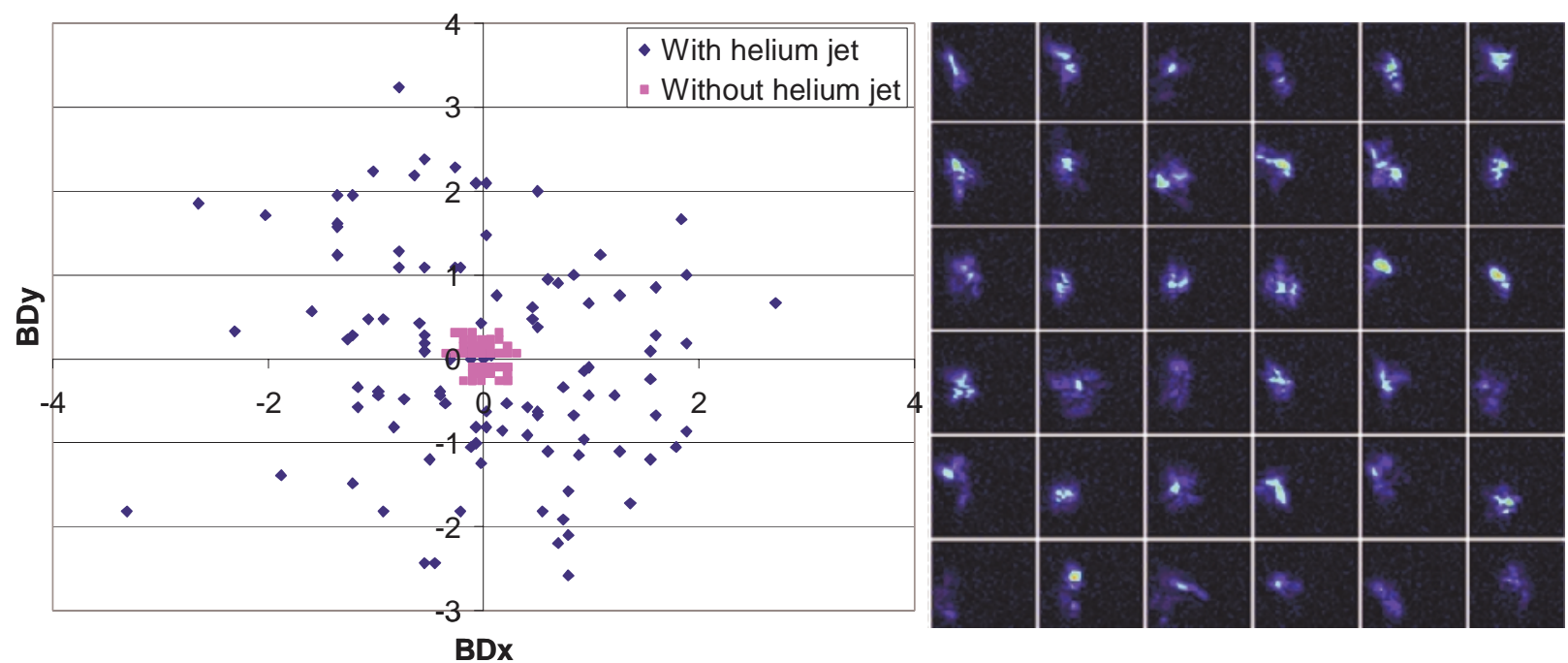

a)

b)

Figure 13 a) Centroid position of the narrow band dye laser with and without the turbulent jet. b) Images of the narrow band dye laser at the focal plane.

Turbulent beam steering is obtained through the device shown in Figure 12. A flow of 107 slpm of helium enters a 1 inch internal diameter, "T" shaped pipe fitting, then bifurcates, generating two jets of helium. The two dye laser beams pass through the fitting. The green beam passes below the fitting and therefore it is only slightly affected by the flow. The device is placed 5 meters before the focusing lens. For this experiment the beam FWHM at the measurement volume were 50, 60 and 120 microns respectively for the green, yellow and red lasers.

Figure 13a) contains plots of the yellow beam centroid position normalized by yellow beam FWHM for 100 shots with and without the jet. The standard deviation of $B D_{y}$ is 0.09 without the jet, 1.24 with the jet. Figure 13b) shows single shots images of the yellow beam at the focal plane. The steering of the beam is clearly visible in this set of pictures, as well as the diffusion effect due to turbulence. The beam appears generally larger and the irradiance distribution is randomly altered. The green beam does not pass through the pipe fitting and is only slightly affected by the helium jet; with no jet the standard deviation of $B D_{y}$ is 0.2 , and with the helium jet is 0.35 . The helium jet affects the green beam only slightly but even in its absence displacements of the green beam should not be neglected.

Five hundred ambient air spectra were collected with and without the helium jet for three

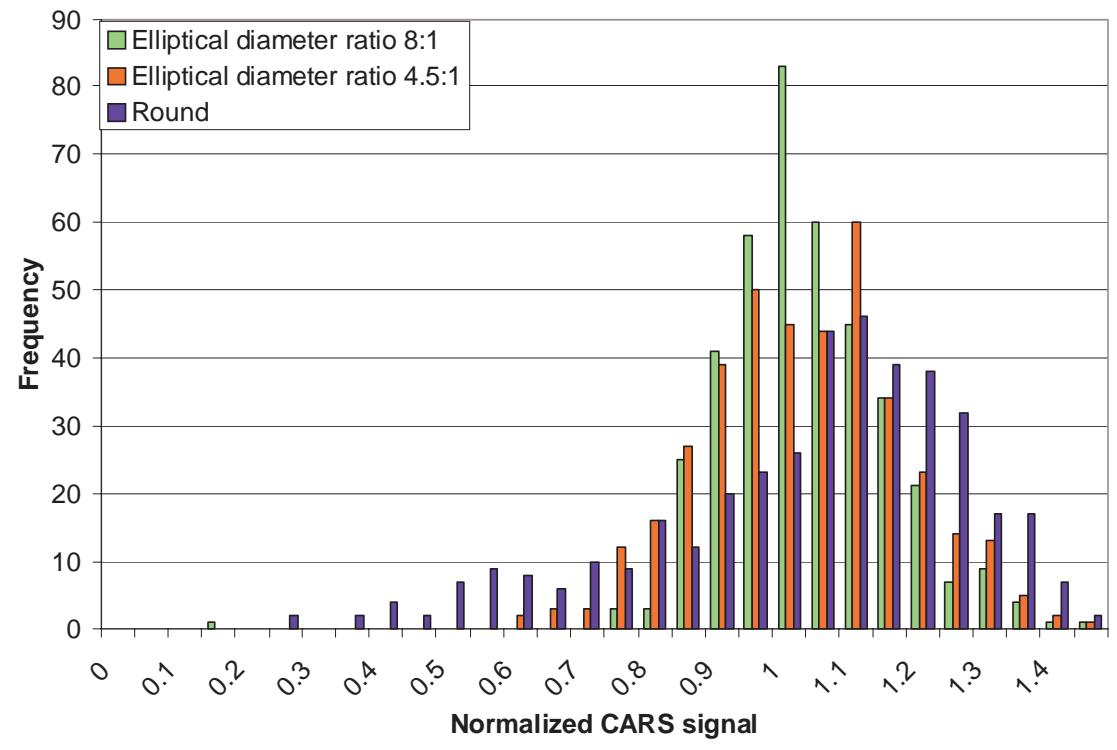

Figure 14. Histogram of CARS signal for various diameter ratios without the helium jet diameter ratios and the signal level evaluated as in the previous experiment. Figure 14 shows histograms of the signal level for the round and two elliptical beams without the helium jet. Signal levels are normalized by the average value. The irradiance of the 
green beam was tuned so that approximately the same signal level was obtained with the elliptical and the round beam. The standard deviation of the signal level distribution is $11 \%$ of the average signal for the elliptical beam of diameter ratio $8: 1$, is $15 \%$ for a $4.5: 1$ diameter ratio and $24 \%$ for the round beam, and for a round beam the histogram is no longer symmetric. The increase in the standard deviation must be caused by beam steering. In fact the beams are aligned for optimum overlap, thus any beam steering will only lower the signal generating an asymmetry in the distribution.

CARS signal intensity is proportional to the product of power of the three laser beams. Relative power fluctuations measurements for each laser were obtained by sending a small portion of the beam to a detector. Five hundred pulses for each beam were collected and analyzed. Measurements for each beam were not simultaneous. Since the green beam energy is a fixed fraction of the beams that pump the two dye lasers we expect some correlation in the power of the three beams. To estimate the effect of laser energy fluctuation on CARS signal the product of the three laser powers is evaluated in the two limit cases of full and no correlation. Physically full correlation implies that the fluctuation in the dye lasers are due exclusively to fluctuations in the pumping beam, no correlations assumes that factors other than the pump energy are predominant. The standard deviation of the product of the three laser powers is $10.6 \%$ of the average for no correlation and $17.6 \%$ for perfect correlation. Figure 15 compares the histogram of CARS signal obtained with a 10:1 diameter ratio beam to the histograms of the product of laser intensities in the two limit cases. The CARS signal histogram lies within the two limit cases suggesting that for the $8: 1$ ratio the fluctuations are due mostly to power fluctuations, and not to beam

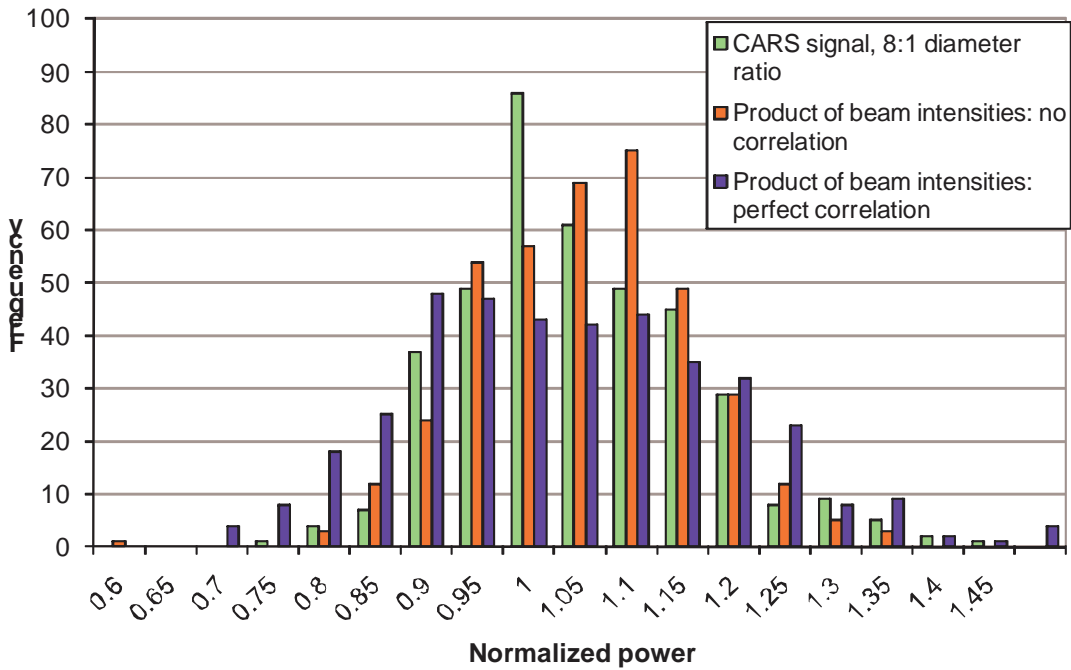

Figure 15. Comparison between the histograms of CARS signal and product of laser intensities

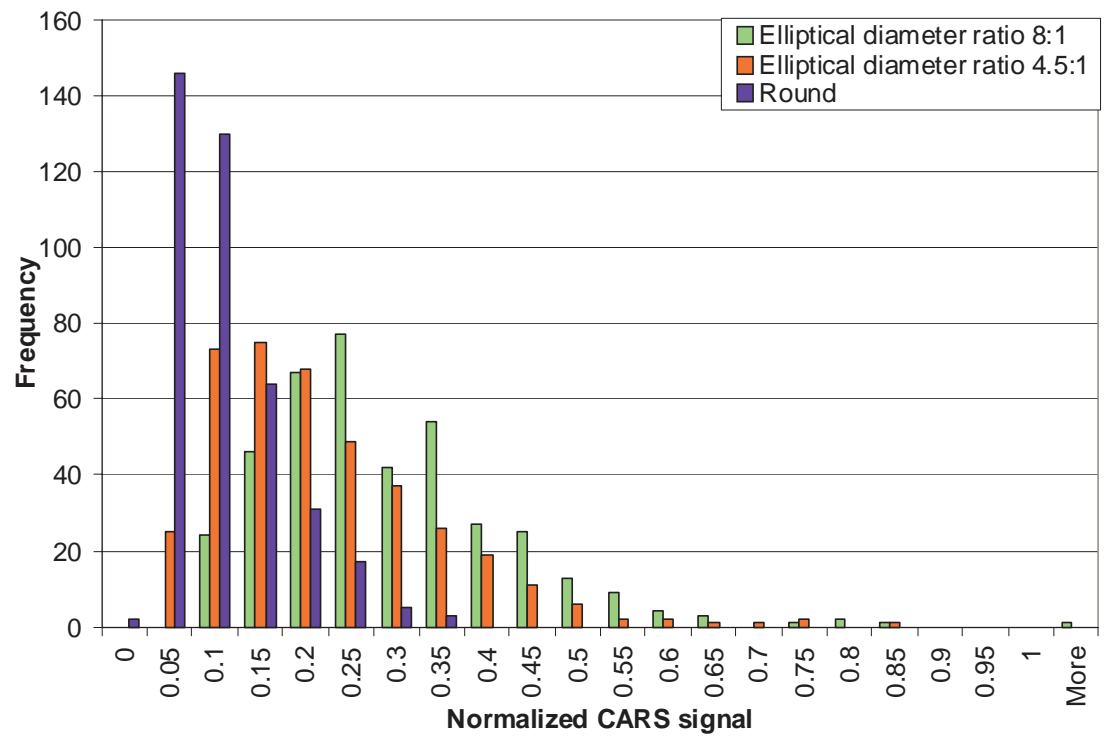

Figure 16. Histogram of CARS signal for various diameter ratios with the helium jet steering.

Figure 16 shows the results obtained with the helium jet flowing. CARS signal level is normalized dividing by the average signal level when the jet is off. The average signal increases with the increase in diameter ratio, going from $8.2 \%$ for the round beam, to $19.5 \%$ for the $5: 1$ ratio, and $26.5 \%$ for the $8: 1$ ratio. The signal level distribution is broader for the elliptical beams; the standard deviations are $0.064,0.126$ and 0.129 for the round, 4.5:1 and 8:1 case respectively. The signal standard deviations as a fraction of mean however, decrease with elliptical beams, and the 
change in the shape of the distributions is similar to the change in shape of a Poisson distribution as the number of samples is increased.

One of the major problems caused by beam steering is the low data yield. When the signal to noise ratio is too low, data have to be rejected. If we assume as 0.1 the threshold below which we reject the data for this experiment, the data yield would be $30.15 \%$ for the round beam, $76.24 \%$ for the $4.5: 1$, and $94 \%$ for the $8: 1$ ratio. The threshold chosen is completely arbitrary, but shows the effectiveness of the technique in increasing the data yield.

\section{Effects of beam diffusion}

In the presence of the helium jet, laser power fluctuations, beam steering, and beam diffusion affect the CARS signal level producing the histogram of Figure 16. Based on the experimental data collected, we can model the effects on the CARS signal of the power fluctuations and of the beam steering, so that we can better understand the effect of the beam diffusion. In an ideal case, where none of these perturbations are present, relative signal level would be constant and equal to 1 . In the presence of power fluctuations, based on the results shown in Figure 14 and Figure 15, the CARS signal level has a Gaussian distribution with average 1 and standard deviation 0.11. According to the results of the beam displacement experiment (see Figure 11), the relative signal level $S_{C A R S}$, in the presence of the beam steering, can be expressed as a Gaussian function in $B D_{y}$ with width $\sigma_{e}$ of 0.6 for the round beam, 1.651 for the 10:1 diameter ratio elliptical beam. For this analysis we neglect the effect of in-plane beam displacement. When both beam steering and power fluctuations are present, the relative signal level can be expressed as

$$
S_{C A R S}=P_{3} \cdot \exp \left(-\frac{2 B D_{y}^{2}}{\sigma_{e}^{2}}\right)
$$

$P_{3}$ takes into account the effect of the random power fluctuation and $B D_{y}$, the beam displacement in the out-of-plane direction. $P_{3}$ and $B D_{y}$ are normal random variables: $P_{3} \sim N(1,0.11)$, and $B D_{y} \sim N(0,1.24)$. The notation $Z=N(\mu, \sigma)$ indicates that the generic random variable $Z$ has a Gaussian distribution with mean $\mu$ and standard deviation $\sigma$. Beam displacement in the $x$ direction is neglected. A numerical experiment has been performed giving input to the Eq. (7) five hundred values of $P_{3}$ and $B D_{y}$ extracted randomly from their respective distributions. The results are representative of what would have been obtained experimentally if the beam diffusion effect was negligible.

Figure 17 shows histograms of the numerical results. For the round beam the average normalized signal is 0.428 , its standard deviation 0.375 , and $32 \%$ of the values are below 0.1 . For the elliptical beam the average is 0.93 , standard deviation 0.14 , and the signal is always above 0.1 . However the average values are lower in the experimental results (Figure 16) because the diffusion makes the beam diameter larger at the focal plane. The reduction of the irradiances of the beams causes a clustering of the data in the lower bins. However the increased size of the beams makes the signal less sensitive to the beam steering. When using a round beam, these two effects combined produce a lower standard deviation relative to the mean in the experimental data than in the computed values. It is interesting to notice that in this experiment beam shaping is even more effective in the presence of beam diffusion. In the absence of beam diffusion the average signal obtained using the elliptical beam is 2.2 times the one obtained with the round beam, but in the experiment it was 3.23 times larger. This result suggests that beam shaping also slightly mitigates the effect of beam diffusion.

Beam diffusion can be added to the numerical model so that we

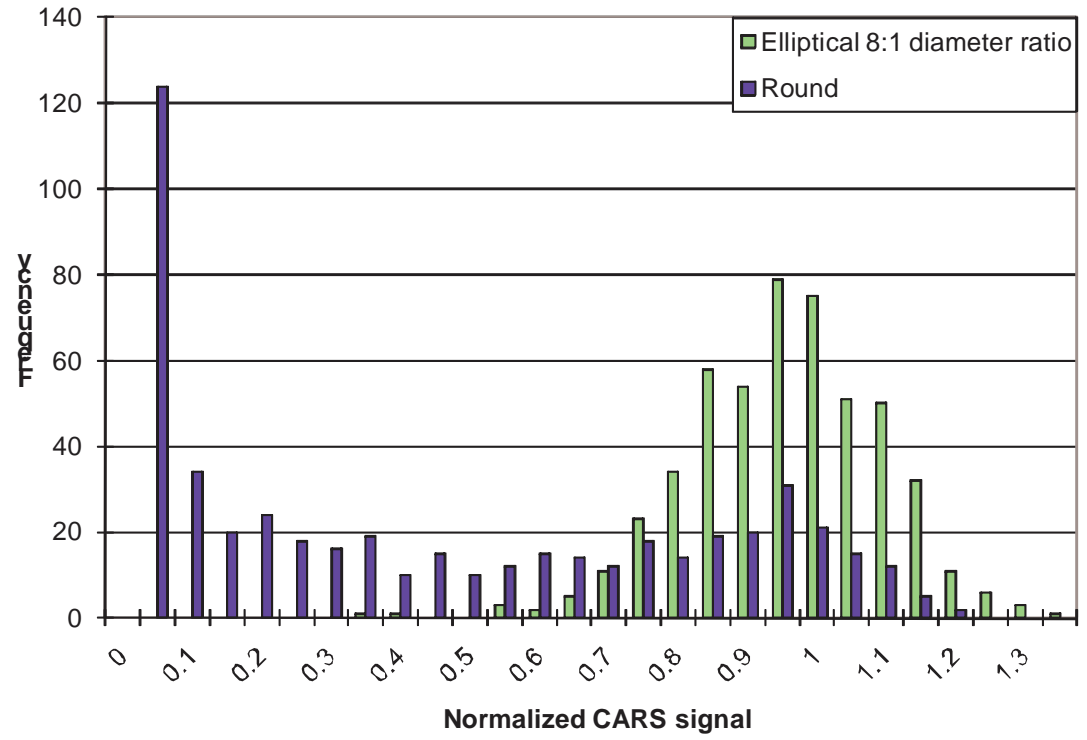

Figure 17. Histograms of CARS signal computed for negligible beam diffusion. 
can simulate the effect of the turbulent helium jet on CARS signal. For each beam we define a diffusion coefficient as the ratio of the beam diameters with and without the turbulent jet flowing

$$
D=\left(D_{x}, D_{y}\right)=\left(\frac{\sigma_{x}^{(\text {turbulent })}}{\sigma_{x}}, \frac{\sigma_{y}^{(\text {turbulent })}}{\sigma_{y}}\right)
$$

For the yellow and red beam we assume that $D_{x} \sim N(1.53,0.27)$ and $D_{y} \sim N(2.86,1.03)$. Average and standard deviation of the diffusion coefficient were evaluated from focal plane images of fifty single shots of the yellow beam taken with the turbulent jet flowing. In the presence of beam diffusion, beam steering and power fluctuations the CARS signal level can be estimated as

$$
S_{C A R S}=P_{3} \cdot P_{C A R S}
$$

$P_{\text {CARS }}$ is evaluated from Eq. (1) using the algorithm of section II. The irradiance of the yellow and red beam is given by Eq. (2) but the parameters $\mu$ and $\sigma$ are modified by beam steering and diffusion respectively:

$$
\begin{gathered}
\mu_{x}=\tan (\alpha)-B D_{x} \cdot d \\
\mu_{y}=B D_{y} \cdot d \\
\sigma_{x}^{(\text {turbulent })}=D_{x} \cdot \sigma_{x} \\
\sigma_{y}^{(\text {turbulent })}=D_{y} \cdot \sigma_{y}
\end{gathered}
$$

$P_{3}, B D_{x}, B D_{y}, D_{x}$, and $D_{y}$ are normal random variables with mean and standard deviations evaluated experimentally.

A numerical experiment has been performed, evaluating the

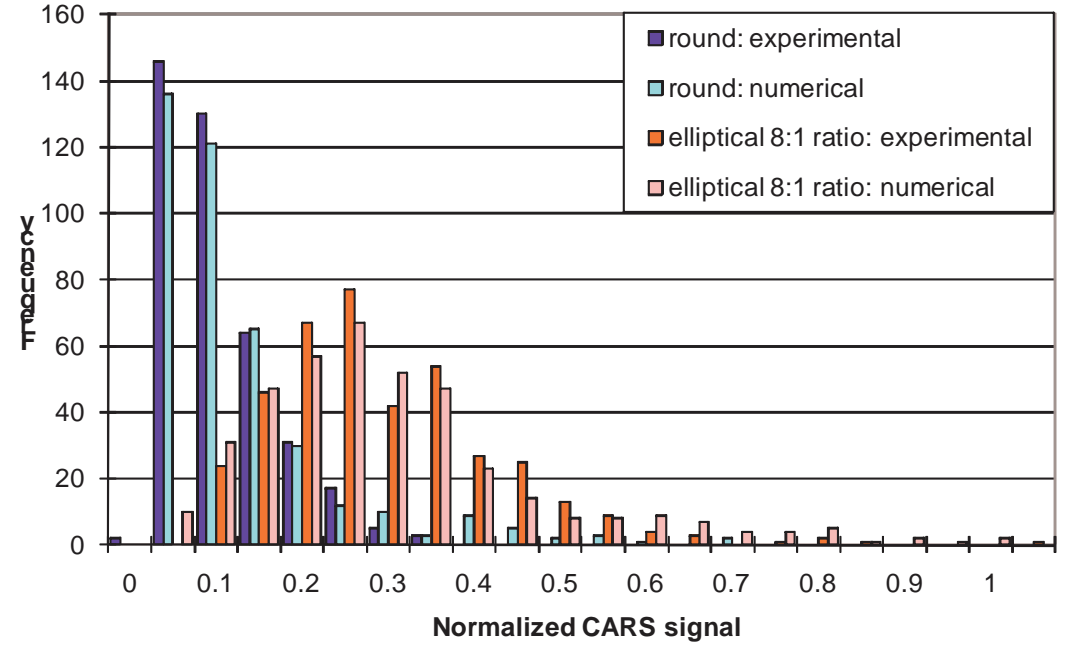

Figure 18. Histograms of CARS signal level with the helium jet from experiments and numerical simulations

CARS signal level for five hundred values of the random variables above. Figure 18 compares the numerical results to the experimental results. The numerical simulation predicts an average signal of 0.1 for the round beam, and 0.28 for the elliptical beam, slightly higher than the measured 0.08 and 0.26 . The standard deviations obtained numerically are 0.11 and 0.16 ( 0.06 and 0.13 experimentally). The discrepancy between the numerical and experimental results is consequence of the simplifying assumptions in the modeling, but it is surprisingly small, given the complexity of the problem. The numerical model can be used to predict the CARS signal level distribution, for any given beam steering conditions and beam diameters.

\section{Conclusions}

A combination of a planar BOXCARS phase matching configuration and shaping of one of the laser beams has been investigated as a tool to mitigate the reduction of CARS signal caused by the refraction of the pump and Stokes beams as they pass through unsteady density gradients produced by turbulent flows. The signal loss is due both to beam steering (relative displacement of the beams) and beam diffusion (increase in the size of the beams at the focal plane). The technique results in a signal that is insensitive to displacement in the plane of the beams, and less sensitive to "out-of-plane" displacement. The spatial resolution is not reduced by this technique. The technique was tested in experiments in which the beams were deflected by movements of a mirror and by a turbulent flow of helium and air, and it proved to be effective in the mitigating the effects of beam steering. The technique also proved to slightly mitigate the loss of signal due to beam diffusion. This approach is recommended for systems in which the irradiance of the shaped beam at the measurement volume is limited by saturation effects and unused energy is available. Future work will implement this technique to measurements in large scale turbulent flows of interest. 


\section{Acknowledgments}

This work was supported by the NASA Fundamental Aeronautics Program, Hypersonics Focus under grants NNX07AC32A and NNX08AB31A (Technical monitors Paul Danehy and Richard Gaffney). The authors would like to thank L.G. Wilson for technical support in performing these experiments.

\section{References}

${ }^{1}$ Cutler A. D., Danehy P. M., Springer R. R., O’Byrne S., Capriotti D. P., and Deloach R., "Coherent Anti-Stokes Raman Spectroscopy Thermometry in a Supersonic Combustor," AIAA J., Vol 41, No 12, Dec 2003, pp. 2451-2459.

${ }^{2}$ Anderson T. J., and Eckbreth A. C., "Simultaneous Coherent Anti-Stokes Raman Spectroscopy Measurements in Hydrogen Fueled Supersonic Combustion,” J. Propul. Power, Vol 8, No1, Jan 1992, pp. 7-15.

${ }^{3}$ Yang S. R., Zhau J. R., Sung G. J., and Yu G., "Multiplex CARS Measurements in Supersonic Hydrogen/Air Combustion," Appl. Phys. B, Vol 68, No 2, 1999 pp 257-265.

${ }^{4}$ Lucht R. P., "Three laser Coherent Anti-Stokes Raman Scattering Measurements of Two Species,” Opt. Lett., Vol 12, 1987 pp 78-80.

${ }^{5}$ O'Byrne S., Danehy P. M., Tedder S. A., and Cutler A. D., "Dual pump Coherent Anti-Stokes Raman Scattering Measurements in a Supersonic Combustor", AIAA J., Vol 45, No 4, April 1997, pp 922-933.

${ }^{6}$ Tedder S. A., Bivolaru D., and Danehy P. M., "Characterization of a Combined CARS and Interferometric Rayleigh Scattering System," 45 ${ }^{\text {th }}$ AIAA Aerospace Science Meeting, Reno NV Jan 2007, AIAA 2007-871.

${ }^{7}$ Danehy P. M., Magnotti G., Bivolaru D., Tedder S. A., and Cutler A. D. "Simultaneous Temperature and Velocity Measurements in a Large Scale Supersonic Heated Jet," 55 ${ }^{\text {th }}$ JANNAF Propulsion Meeting, Boston, MA, 2008, Paper 1193.

${ }^{8}$ Tedder S. A., Danehy P.M., Magnotti G., and Cutler A.D., "CARS Temperature Measurements in a Combustion Heated Supersonic Jet," $47^{\text {th }}$ AIAA Aerospace Science Meeting, Orlando, FL, Jan 2009, AIAA 2009-524.

${ }^{9}$ Eckbreth A. C., Laser Diagnostics for Combustion Temperature and Species, $2^{\text {nd }}$ ed. Gordon and Breach Publishers, 1996.

${ }^{10}$ Herring G. C., Hart R. C., Fletcher M. T., Balla J. R., Henderson B.S., "Prospects for non linear diagnostics in the jet noise laboratory", NASA/TM 2007-214893

${ }^{11}$ Eckbreth A. C., Dobbs G. M., Stufflebeam J.H., and Tellex P.A., "CARS Temperature and Species Measurements in Augmented Jet Engine Exhausts," Applied Optics Vol. 23 No. 9, 1984, pp1328-1339.

${ }^{12}$ Greenhalgh D. A., "Comments on the use of BOXCARS for Gas-Phase CARS Spectroscopy," Journal of Raman Spectroscopy, Vol 14 No.3, 1983 pp 150-154.

${ }^{13}$ Teets R. E., "CARS Signals: Phase matching, Transverse Modes, and Optical Damage Effects," Applied Optics Vol. 25, No. 6, 1986, pp 855-862.

${ }^{14}$ Hecht E., Optics $3^{\text {rd }}$ Ed, Addison Wesley Longman Publishers.

${ }^{15}$ D. Bivolaru, C.G. Herring, "Focal plane imaging of crossed beams in non-linear optics experiments", Rev. Sci. Instrum., Vol 78, 2007 